INPLASY

PROTOCOL

To cite: Deng et al. Effect of

lateral wedge-shaped

orthopedic insole on patients

with genu varus: A protocol for

systematic review and meta-

analysis. Inplasy protocol

202190002. doi:

10.37766/inplasy2021.9.0002

Received: 01 September 2021

Published: 01 September 2021

Corresponding author:

Zhongyi Deng

437096903@qq.com

Author Affiliation:

School of Sports Medicine and Health, Chengdu Sport

University.

Support: None.

Review Stage at time of this submission: Preliminary

searches.

Conflicts of interest:

None declared.

\section{Effect of lateral wedge-shaped orthopedic insole on patients with genu varus: A protocol for systematic review and meta-analysis}

Deng, ZY1; Wu, GT2; Luo, HQ³ .

Review question / Objective: The objective of this systematic review was to determine the therapeutic effect of lateral wedge orthotic insoles on patients with varus genu.

Condition being studied: Orthopaedic insole is foot biomechanics expert Dr. Merton put forward, corrective insole is currently the most common prevention of foot diseases, correct the shape of the foot, maintain the health of the foot auxiliary appliances. Genu varus is a common bad posture. Patients with long-term genu varus may have knee pain symptoms. The main complaints of patients are genu varus deformity (O-leg) and knee joint flare-up or persistent pain. According to the biomechanical analysis of human body, when genu varus malformation exists, the strength will be conducted too much from the inside of the knee joint, leading to excessive bearing and accelerated wear of the cartilage on the inside articular surface of the knee joint, resulting in premature osteoarthritis. The purpose of this study was to evaluate the correction effect of lateral wedge orthotic insoles in patients with genu varus.

INPLASY registration number: This protocol was registered with the International Platform of Registered Systematic Review and Meta-Analysis Protocols (INPLASY) on 01 September 2021 and was last updated on 01 September 2021 (registration number INPLASY202190002).

\section{INTRODUCTION}

Review question / Objective: The objective of this systematic review was to determine the therapeutic effect of lateral wedge orthotic insoles on patients with varus genu.
Condition being studied: Orthopaedic insole is foot biomechanics expert $\mathrm{Dr}$. Merton put forward, corrective insole is currently the most common prevention of foot diseases, correct the shape of the foot, maintain the health of the foot auxiliary appliances. Genu varus is a 
common bad posture. Patients with longterm genu varus may have knee pain symptoms. The main complaints of patients are genu varus deformity (O-leg) and knee joint flare-up or persistent pain. According to the biomechanical analysis of human body, when genu varus malformation exists, the strength will be conducted too much from the inside of the knee joint, leading to excessive bearing and accelerated wear of the cartilage on the inside articular surface of the knee joint, resulting in premature osteoarthritis. The purpose of this study was to evaluate the correction effect of lateral wedge orthotic insoles in patients with genu varus.

\section{METHODS}

Participant or population: All genu varus patients regardless of age, sex, race, country or disease.

Intervention: The experimental group wore the outer wedge orthopedic insoles.

Comparator: The control group received a placebo or other treatment technique.

Study designs to be included: RCTs.

Eligibility criteria: All randomized controlled trials (RCTs) reported will be included without regional and language restrictions. Animal studies, cohort studies, casecontrolled studies, case reports, and expert experience will be excluded.

Information sources: The following electronic databases will be searched from inception to December 2020: PubMed, the Cochrane Library, Embase, Web of Science, CNKI, WanFang Data, Weipu Electronics. In addition, reference lists of the included studies were manually searched to identify additional relevant studies.

Main outcome(s): VAS pain scale; Plantar pressure analysis; Gait analysis.

Quality assessment / Risk of bias analysis: This study was independently searched by two researchers. They independently screened titles and abstracts for eligibility and read full texts independently to extract data. In case of disagreement, consensus was reached through discussion. Modified Jadad scale (total score is seven, higher score is better quality) was used to estimate the quality of the included RCTs, of which one to three were classified as low-quality, and four to seven were classified as high-quality.

Strategy of data synthesis: XThe metaanalysis will be performed posthoc when an adequate number of sufficiently homogeneous studies are found after data extraction, using Review Manager software. Aggregate-level data will be used for meta-analysis. Data will be pooled using the random-effects model. Continuous outcomes measured on the same scale will be synthesized using mean difference and reported as a mean difference with a $95 \%$ confidence interval (Cl). The Review Manager (RevMan) V.5.4 software will be used for statistical analysis.

Subgroup analysis: Subgroup analysis of study design will be conducted if there is statistical heterogeneity among studies.

Sensitivity analysis: We will perform sensitivity analysis based on sample size, research design, heterogeneity quality, methodological quality, and statistical model, excluding trials with low quality, and ensure the stability of analysis results.

Country(ies) involved: China.

Keywords: Outer wedge orthopedic insoles; In the knee; The pain; Biomechanical analysis

Contributions of each author:

Author 1 - Zhongyi Deng.

Email: 437096903@qq.com

Author 2 - Gaitian Wu.

Email: 714395069@qq.com

Author 3 - Huiqiang Luo.

Email: 495032653@qq.com 